\title{
Instantaneous frequency estimation of non-stationary signal
}

\author{
Qi Xiaoxuan ${ }^{1, a^{*}} \quad$ Guo Tingting ${ }^{2, b}$ \\ ${ }^{1}$ School of Electrical Engineering \& Automation, Tianjin University, China \\ ${ }^{2}$ School of information engineering, Shenyang University, China \\ aqi_xx@aliyun.com bytuguoting@163.com
}

Key words: instantaneous frequency; hilber transformation; teager energy operator

Abstract: In signal processing, the estimation of instantaneous frequency is a kind of very important issue for non-stationary multi-component signal. Firstly, this paper adopts EMD decomposition to decompose non-stationary multi-component signal and convert into a series of single component signal. Then using the method of hilber transform and teager energy operator respectively, the instantaneous frequency of single component can be obtained. Finally, the simulation results show that adopting hilber transform can make root-mean-square error of instantaneous frequency and amplitude of fluctuation more smaller. So this method presented in the paper is feasible and effective.

\section{Introduction}

Instantaneous frequency estimation of non-stationary signal is a very important physical quantity, it is estimated instantaneous frequency of the signal processing in many practical applications ${ }^{[1-2]}$. Traditional time-frequency analysis method is not reflect the signal therefore has no validity for analysis of non-stationary signal ${ }^{[3]}$. This paper adopts empirical mode decomposition (EMD) decomposition to decompose non-stationary multi-component signal and convert into a series of single component signal, then respectively adopt the method of hilber transform method and teager energy operator for each single component of the decomposed signal instantaneous frequency estimation. Finally, the simulation results show that adopting hilber transform can make root-mean-square error of instantaneous frequency and amplitude of fluctuation more smaller. So this method presented in the paper is feasible and effective.

\section{Instantaneous frequency estimation}

\section{Empirical mode distribution}

From a physical point of view, the signal can be divided into single component signal. According to the definition of instantaneous frequency multiplexed signal only when the signal is a single component. But in fact, directly receive and observed signals are more real signal component, so component of the complex signal is decomposed into a single component ${ }^{[4]}$.

EMD is obtained by non-linear and non-stationary signal decomposition to obtain a series of characterization of signal characteristic time scales of a modal function intrinsic (IMF), makes the individual IMF is a single component amplitude or frequency modulation signal, so that the definition of instantaneous frequency of physical significance ${ }^{[5]}$. For a time series $x(t)$ after EMD can get 


$$
x(t)=\sum_{i=1}^{n} c_{i}(t)+r_{n}(t)
$$

Decomposed by the EMD method for each single component of the AM-FM signal $c_{i}(t)$ respectively hilber transform and teager energy operator is used to obtain instantaneous frequency.

\section{Hilber transform}

By the EMD method resulting from the decomposition of each single component signals based on hilbert transform structure, then according to the analytical signal instantaneous frequency.

Formula (1) each intrinsic mode function $c_{i}(t)$ for the hilbert transform

$$
H\left[c_{i}(t)\right]=\frac{1}{\pi} \int_{+\infty}^{-\infty} \frac{c_{i}(\tau)}{t-\tau} d \tau
$$

Phase function

$$
\theta_{i}(t)=\arctan \left[\frac{H\left[c_{i}(t)\right]}{c_{i}(t)}\right]
$$

Get instantaneous frequency $w_{i}(t)$

$$
w_{i}(t)=\frac{d \theta_{i}(t)}{d t}
$$

Because the resulting from the empirical mode decomposition of intrinsic mode function is single component signals, thus ensure the type (4) has a physical meaning.

\section{Teager energy operator}

Teager energy operator is usually referred to as $\psi$, For continuous signal $x(t)$, which operation can be expressed as $\psi$ :

$$
\psi[x(t)]=[x(t)]^{2}-x(t) x(t)
$$

where $x(t)=\frac{d x}{d t}, x(t)=\frac{d^{2} x}{d t^{2}}$ 。It can be obtained by solving the instantaneous frequency $f_{i}(t)$

$$
f_{i}(t)=\sqrt{\frac{\psi[x(t)]}{\psi[x(t)]}}
$$

\section{Simulation Analysis}

The following two simulation examples are given to illustrate the effectiveness of the two methods. Simulation examples given $x(t)$ of LFM signal, its expression is as follows:

$$
x(t)=x_{1}(t)+x_{2}(t)
$$

among them $x_{1}(t)=\cos \left[2 \pi\left(5 t^{2}+2 t\right)\right], x_{2}(t)=\cos \left[2 \pi\left(50 t^{2}+20 t\right)\right]$

The instantaneous frequency of the signal is 


$$
f_{1}(t)=10 t+2
$$

$$
f_{2}(t)=100 t+20
$$

The signal $x(t)$ time domain waveform is shown in Fig. 1.

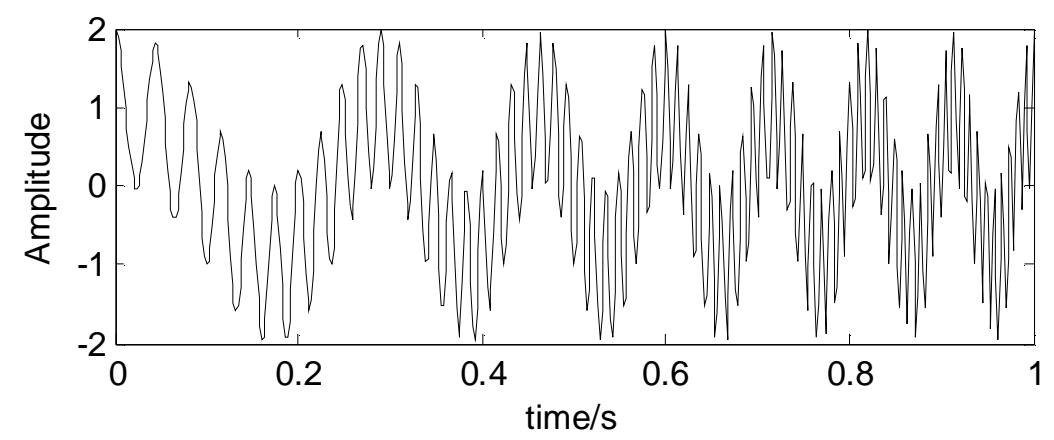

Fig. 1 The simulation signal $x(t)$ time domain waveform

At this point the simulated signal $x(t)$ is two chirp signals and form, this multi-component signal EMD decomposed each single component signal and forms,as shown in Fig.2.Thus, multi-component signal it can be decomposed into a set of single component signals.

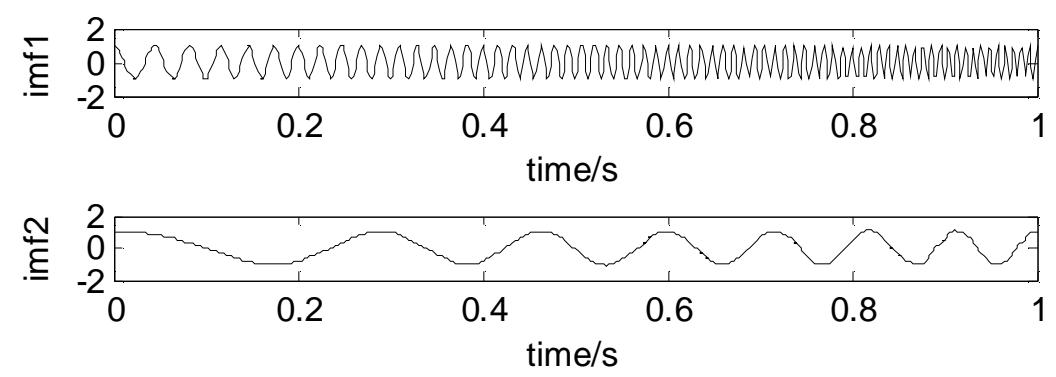

Fig. 2 The results of the simulation signal $x(t)$ of EMD

In order to more accurately estimate the instantaneous frequency of the signal using the least squares method to estimate the instantaneous frequency after fitting, as Fig. 3. Fig. 3 shows the use of the instantaneous frequency hilber transform estimation curve obtained with the actual instantaneous frequency signal curve is coincident, further evidence of the effectiveness.

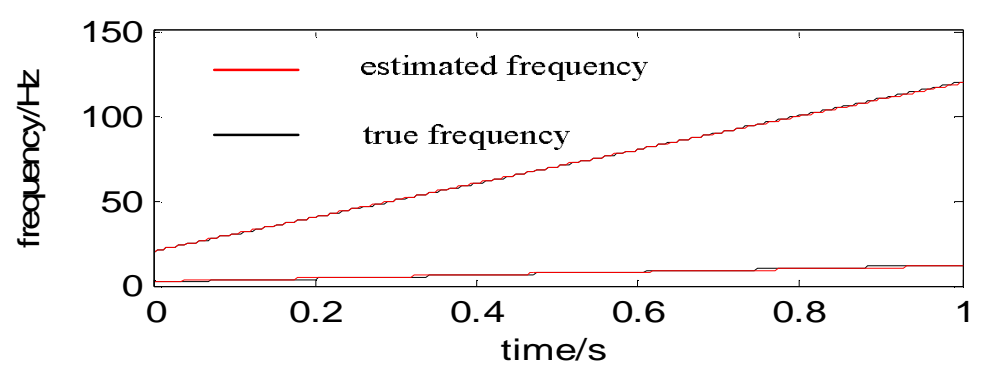

Fig. 3 Hilber request signal conversion method using each component of $x(t)$ instantaneous frequency curve fitting 
The instantaneous frequency estimate obtained using teager method were fitted using the least squares method, as shown in Fig. 4. From the curve in Fig. 4 the instantaneous frequency estimate and the actual original signal instantaneous frequency deviation curve at higher frequencies.

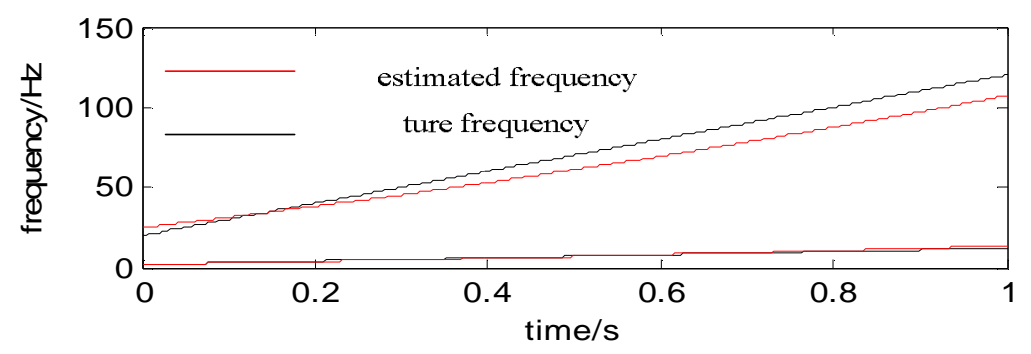

Fig. 4 Teager method using energy operator request signal $x(t)$ each component of

the instantaneous frequency curve fitting

In order to better observe the two methods required to verify the accuracy of the instantaneous frequency, instantaneous by calculating the root mean square error of each frequency component to verify the results shown in Table 1 . Table 1 shows hilber transformation method for the simulation of signal instantaneous frequency of the root mean square error is smaller than teager energy operator .

Table 1 two methods to calculate the instantaneous frequency of root mean square error

\begin{tabular}{|l|l|l|}
\hline Root mean square error & Hilber Transform & Teager Energy Operator \\
\hline imf1 & 0.2581 & 0.4540 \\
\hline imf2 & 0.2785 & 9.1069 \\
\hline
\end{tabular}

Simulation analysis of the appeal does not contain the signal noise, but the real signal is generally subject to interference noise, to appeal the simulated signal to noise ratio is $10 \mathrm{~dB}$ plus Gaussian white noise. For the data in Table 2 shows that hilber transformation method for the simulation of signal instantaneous frequency of the root mean square error is smaller than teager energy operator .

Table 2 Two methods including $10 \mathrm{db}$ gaussian white noise of the instantaneous frequency of the root mean square error

\begin{tabular}{|l|c|l|}
\hline Root mean square error & Hilber Transform & Teager Energy Operator \\
\hline imf1 & 3.3801 & 23.9464 \\
\hline imf2 & 31.1682 & 37.4382 \\
\hline
\end{tabular}

The simulation results show that using the EMD method can realize the decomposition of the multi-component signal into a signal and the form of a single component. Respectively, using hilber transform method of calculating the instantaneous frequency is better.

\section{Conclusion}

Instantaneous frequency estimation of non-stationary signal is a very important physical quantity, from which we can know that the law of the signal frequency changes over time, hold a key method is significance. Instantaneous frequency estimation of definitions for a single component signals, this paper adopts EMD decomposition to decompose non-stationary multi-component signal and convert into a series of single component signal. Then using the method of hilber transform and teager energy operator respectively, the instantaneous frequency of single component can be 
obtained. Trough the simulation experiment shows the hilber transform method presented in the paper is feasible and effective.To be sure, when nonstationary multi-component complex and contains noise, the method effect is not ideal enough, needs to be further research.

\section{References}

[1] $\mathrm{Xu}$ Lingjing. Matched wigner transform and its application to instantaneous frequency estimation[J].Acta electronicasinica,2014,42(11): 2247-2252.

[2]Shi Zhensheng, Yang Yingxin. Harmonic analysis and application based on instantaneous frequency estimation[J]. I.C.E\& Powerplant, 2014,27(2): 125-128.

[3] GuoShaokun.ComParativs study of instantaneous frequency estimation methods[J]. Theory and methods, 2010,29(6): 21-25.

[4]Dai Haomin. Comparative research and improvement on the calculation method of instantaneous frequency[J]. Journal of Sichuan university,2014,51(6):1197-1203.

[5]Liu Jian,Peng Fuqian. A New Order Tracking Method Based on Chirplet Path Pursuit is Application to Gearbox Fault Diagnosis[J]. Journal of mechanical engineering,2009,45(7):81-86.

[6]Li Hui. Order Tracking of Speed - up Signal for Gearbox Based on Instantaneous Frequency Esti mation [J]. Journal of Vibration,Measurement \&Diagnosis, 2007,27(2): 125-128.

[7]Orovic I,Orlandic M,Stankovic Setal.A virtual instrument for time-frequency analysis of signals with highly nonstationary instantaneous frequency[J].IEEE Transaction on instrumentation and measurement,2011,60(3) : 791-803.

[8] Xiumei,Bi Guoan,Stankovic S,et al.Local polynomial Fourier transform: A review on recent developments and applications [J] .Signal Processing,2011,91( 6) : 1370 -1393. 